\title{
Constitutive Relation of Flexible Woven Composites Based on Neural Network
}

\author{
Minjun Gao ${ }^{1}$, Junhui Meng ${ }^{1 *}$, Moning $\mathrm{Li}^{1}$, Nuo $\mathrm{Ma}^{1}$ \\ ${ }^{1}$ School of Aerospace Engineering, Beijing Institute of Technology, Beijing, China \\ *Email: mengjh@bit.edu.cn
}

\begin{abstract}
Due to the advantages of light weight, low cost, foldable and flexible control, flexible inflatable structure has important application prospect in the field of aerospace. Airbags of inflatable structures are usually made of flexible fiber woven composites, which are laminated or heat sealed by load-bearing layer of woven fibers and other functional membrane layers. Study on the flexible fiber woven composites' mechanical property is very important, but it's greatly influenced by environmental parameters like temperature, which results to complex nonlinearity. However, there are many factors influencing the constitutive relation of flexible airbag materials, including Coefficient of friction between fiber yarns, yarn diameter, space, weaving way environmental temperature, loading velocity and other parameters. Therefore, in order to obtain accurate constitutive models of different kinds of airbag materials, a large number of experimental tests are usually required. In this paper, the neural network simulation is combined with the constitutive model construction of flexible airbag materials, a back propagation neural network based constitutive relation analytical method was proposed for the flexible fiber woven composites. The biaxial tensile test was carried out under different environmental parameters to obtain the coupling relationship between shear modulus and biaxial tensile modulus. The experimental results were taken as input variables of the neural network to construct an extended neural network model considering the shear effect and loading history effect. The complex constitutive relation of flexible fiber woven composites was completed by using the advantage of neural network in dealing with nonlinear problems. The constitutive relation obtained from the training was applied to the nonlinear finite element simulation analysis of the flexible inflatable aerostat's deformation under different light conditions in one day. Compared with the experimental results, the prediction accuracy was greater than 0.99 , which verified the validity of the constitutive model..
\end{abstract}

Keywords: Constitutive Relation; Neural Network; Flexible Woven Composite; Complex Nonlinearity 